Original Contribution

\title{
THE CONTROL OF DEAD SPACE WITH ANTIBIOTIC LOADED CEMENT BEADS AND NAILS IN HIGH ENERGY TRAUMA
}

\author{
T. Al-Sadek ${ }^{1}$, A. Al-Sadek ${ }^{2}$, G. Dimitrov ${ }^{3}$, K. Marinov ${ }^{4}$, L. Al-Sadek ${ }^{5}$ \\ ${ }^{1}$ Department of Orthopaedics and Traumatology, Belhoul European Hospital, Dubai, UAE \\ ${ }^{2}$ Medical University, Sofia, Bulgaria \\ ${ }^{3}$ Department of Orthopedics and Traumatology, Medical University, Pleven, Bulgaria \\ ${ }^{4}$ Department of Special Surgery /Thoracic Surgery, Vascular Surgery, Pediatric Surgery and \\ Orthopedics and Traumatology, University Hospital "Prof. Dr. St. Kirkovich", Stara Zagora, Bulgaria \\ ${ }^{5}$ Department of Dermatology and Venereology, University Hospital "Prof. Dr. St. Kirkovich", \\ Stara Zagora, Bulgaria
}

\begin{abstract}
Purpose: To treat delayed present (more than 24h) and Gustilo type III fractures in a better way than external fixation which before was a routine in our hospital.

Methods: We have applied advanced trauma life support control (ATLS). After proper debridement of the wound, irrigation with saline and antibiotic cover, we reduced the fracture with the Sign IM nail, which is a solid nail without internal dead space, and then we added antibiotic loaded cement with $3 \mathrm{~g}$ of Amikacin and $4 \mathrm{~g}$ of Vancomycin.

Results: Out of 38 delayed present fractures, we have observed serous discharge in 3 cases which have been controlled by changing the antibiotic loaded cement. While the other cases remained uninfected.

Conclusion: The best way to deal with infection is to prevent it, while other factors as obesity, diabetes, and other co-morbidities increase the risk of infection. In our method, we also provided local high concentration of antibiotic with the use of cement loaded antibiotic, and the control of dead space with bone defect and tissue loss. If we do not put cement or other solid material, then blood will definitely take space and that would be a media for infection.
\end{abstract}

Key words: Control, Dead Space, Antibiotic, Loaded, Cement, Beads, Nails.

\section{INTRODUCTION}

In 1970, Buchholz and Engelbrecht (1) incorporated Gentamicin in bone cement for the treatment of infection in prosthetic joints. Antimicrobial loaded bone cement (ALBC) has since been used in the treatment of softtissue infections, open fractures, long bone osteomyelitis, and arthroplasty infections.

The use of antibiotic-impregnated cement to deliver high concentrations of antibiotic locally without systemic absorption has been described for infected non-unions in both lower and upper extremities as well as in revasion arthroplasty (3). In treating infections, local antibiotic elution efficacy is far more important than the mechanical strength of the cement, and liquid antibiotics have been shown to provide elution rates than those of powder antibiotics.

\footnotetext{
*Correspondence to: Tabet Al-Sadek, M.D, Ph.D, Belhoul European Hospital, Dubai-UAE. Email: drthabet@abv.bg; Mobile: +971551503964
}

Antibiotics used with bone cement: Amikacin, Amoxicillin, Bacitracin, Cefamindol, Ceftazidime, Cefuroxime, Cefozonam, Cephalothin, Ciprofloxacin, Clindamycin, Colistin, Daptomycin, Erythromycin, Gentamycin, Lincomycin, Methecillin, Tobramycin, Vancomycin, Ticarcillin, Oxacillin $(2,4,5)$.

The use of Aminoglygoside antibiotic Gentamycin and Tobramycin has been established $(2,4,5)$. The use of Vancomycin has gained popularity because of its effectiveness against Methcillin resistant bacteria including Staphylococcus Aureus, and Methcillin resistant Staphylococcus Epidermidis. The optimal antibiotic dosage per 40 gram-bag of cement has not yet been determined. The reported doses of Gentamycin, Tobramycin and Vancomycin range from 2 to $5,2.4$ to $9.6 \mathrm{~g}$ and 3 to $9 \mathrm{~g}$ respectively $(2,4,5)$. 


\section{OBJECTIVES OF THE STUDY}

Purpose of the study was to treat delayed present (more than 24h) and Gustilo type III fractures a better way than external fixation which before was a routine in our hospital.

Defect in high-energy trauma is space for blood and media of infection if not controlled by using antibiotic loaded cement, and protect healing factors from infection and instability.

\section{MATERIALS AND METHODS}

This is a 4-year study from April 2002 to May 2006, in Shifa Hospital in Gaza strip in the Palestinian Territories.

Defects in high energy trauma is an easy space for blood and are more prone to infections if not controlled by using antibiotic loaded cement (Figure 1).

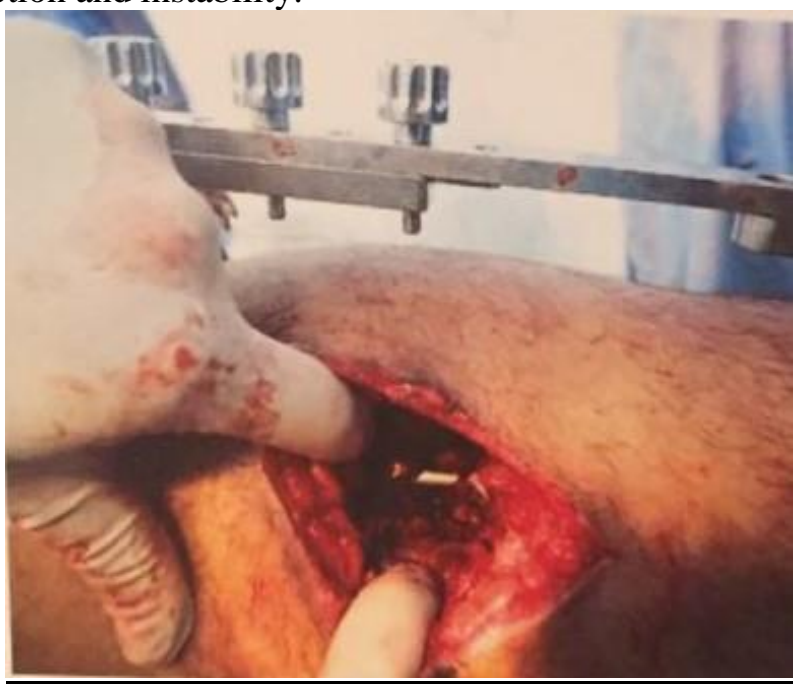

Figure 1. Soft Tissue dead space control in high energy fractures.

From one side we prevent blood collection, and from the other side it's a good place to provide antibiotic to the fracture and the fixation implant, to prevent further infections. It is applied to $1 / 3$ of one side of bone circumference but not around the whole bone. Most of the fracture fragments and healing factors are being protected.

Prior to the orthopedic procedures, we have applied advanced trauma life support control (ATLS). After proper debridement of the wound, irrigation with saline and antibiotic cover, we reduced the fracture with the Sign
IM nail, which is a solid nail without internal dead space, and then we added antibiotic loaded cement with $3 \mathrm{~g}$ of Amikacin and $4 \mathrm{~g}$ of Vancomycin. The amount of cement applied depended on the defect of the bone, and soft tissue loss, without leaving any space after skin closure.

We controlled dead space and lost soft tissue and bone defect and fragments by keeping most of the fragments and space inside the nail canal by a solid Sign IM nail. To control the infection, we limited the space for blood collection by putting antibiotic loaded bone cement beads in the affected part.
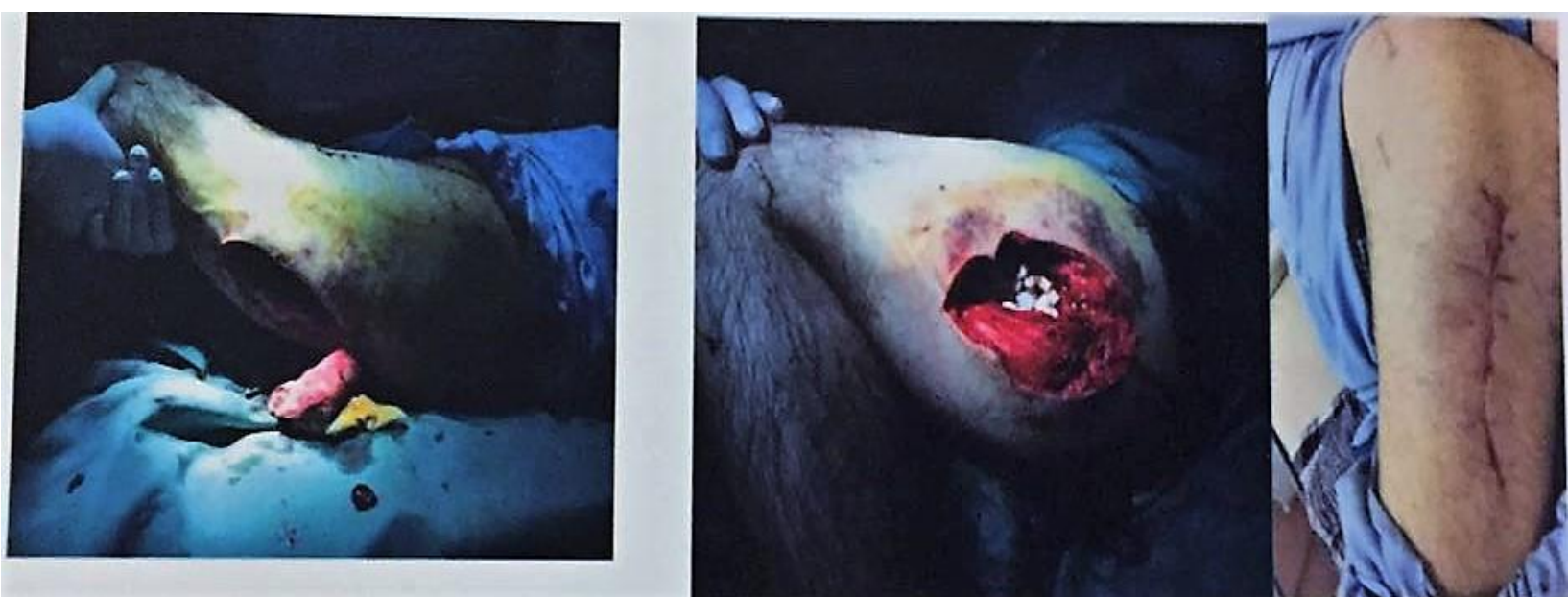

Figure 2. We put cement beads closed to the nail to penetrate the canal if it is exposed. 


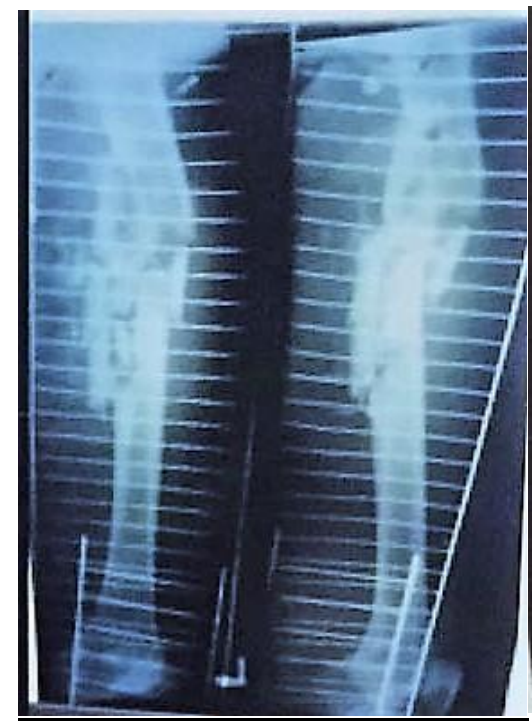

(a)

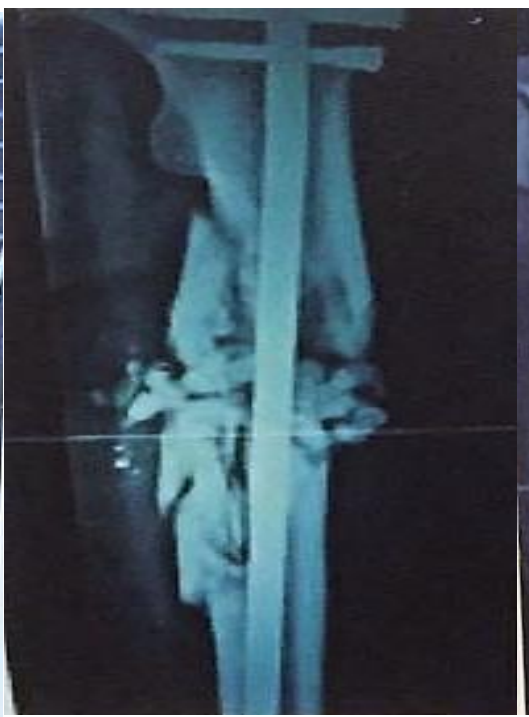

(b)

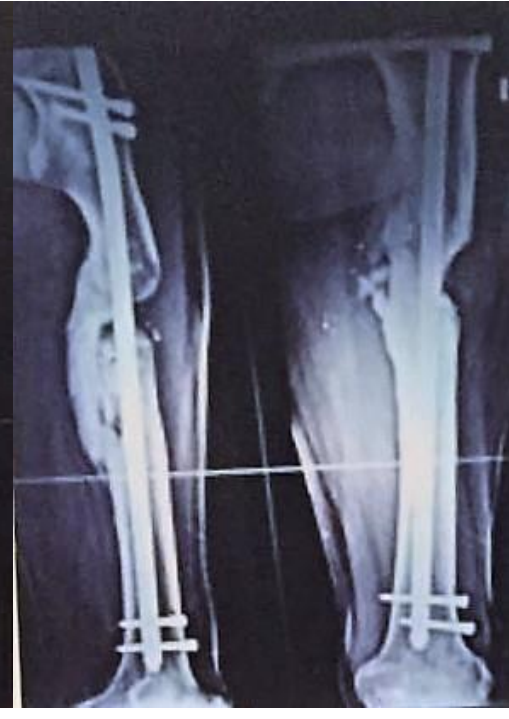

(c)

Figure 3. a- Diagnostic X-ray, b- Post Op X-ray, c- 6 months control X-ray.

\section{RESULTS}

We applied cement-loaded antibiotic and Sign IM nails in 55 cases, 44 of them were gunshot injuries and bomb blast injuries.

Most of the fractures in gunshot injuries were comminuted fractures, with different degree of commination (Figure 3-a), 50\% had more than $4 \mathrm{~cm}$ of cortical damage in both fractures.

Fifteen cases were infected ( 3 post IM Sign nail infection, and 12 non-union with osteomyelitis change in X-rays. 1 was pathological fracture and 11 were post implant infection non-union.

Ages vary between 18 years old and 60 years old with an average age of 30 years old. 43 $(77.18 \%)$ of the cases were Femurs, and 12 (21.81\%) cases were Tibia. All of them were fixed by Sign IM nails (Figure 3-b), and the fractures were controlled by an X-ray after 6 months (Figure 3-c). 47 were males and 3 were female patients.
The surgery was not difficult in delayed present fracture due to the presence of a wound at the fracture site (open fracture).

With time, when cement loss their antibiotic they work as a foreign body, thus a delayed reinfection was noted in the cement site in 2 of the cases.

Out of 38 delayed present fractures, we have observed serous discharge in 3 cases which have been controlled by changing the antibiotic loaded cement. While the other cases remained uninfected.

On follow ups of 6 weeks, 3 months, 6 months and 1 year, we have lost 2 cases in follow up, one non-union which was successfully treated with iliac bone graft and the rest had complete healing and bone union without the need for graft as (Figure 4) below Shows.

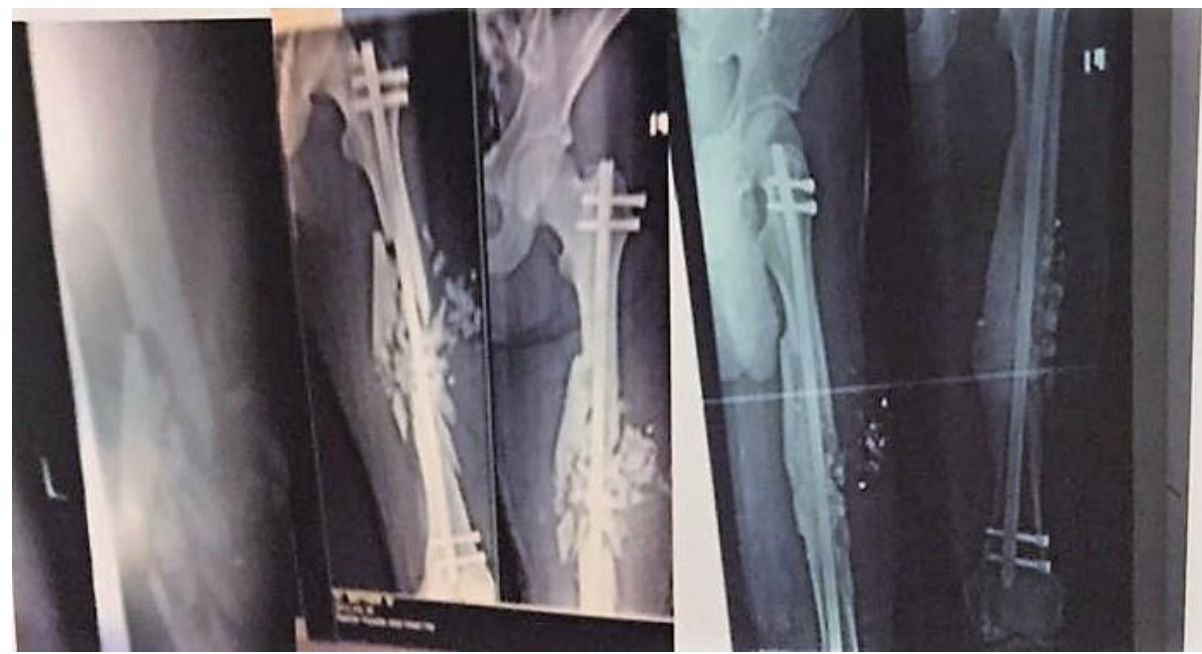

Figure 4. X-Ray showing how we reduced the need for bone graft.

Trakia Journal of Sciences, Vol. 15, № 3, 2017 
In 15 infected non-union result were different and we had to change beads in 7 cases twice, and we did bone graft in 3 cases, in one case we noted a delayed reactivation of infection after healing in cement beads site, and for this reason we removed the cement after 6 weeks in all the infected non-union. We noted healing in infected fracture with improvement in density of infected focus as well in 11 infected cases (Figure 5).
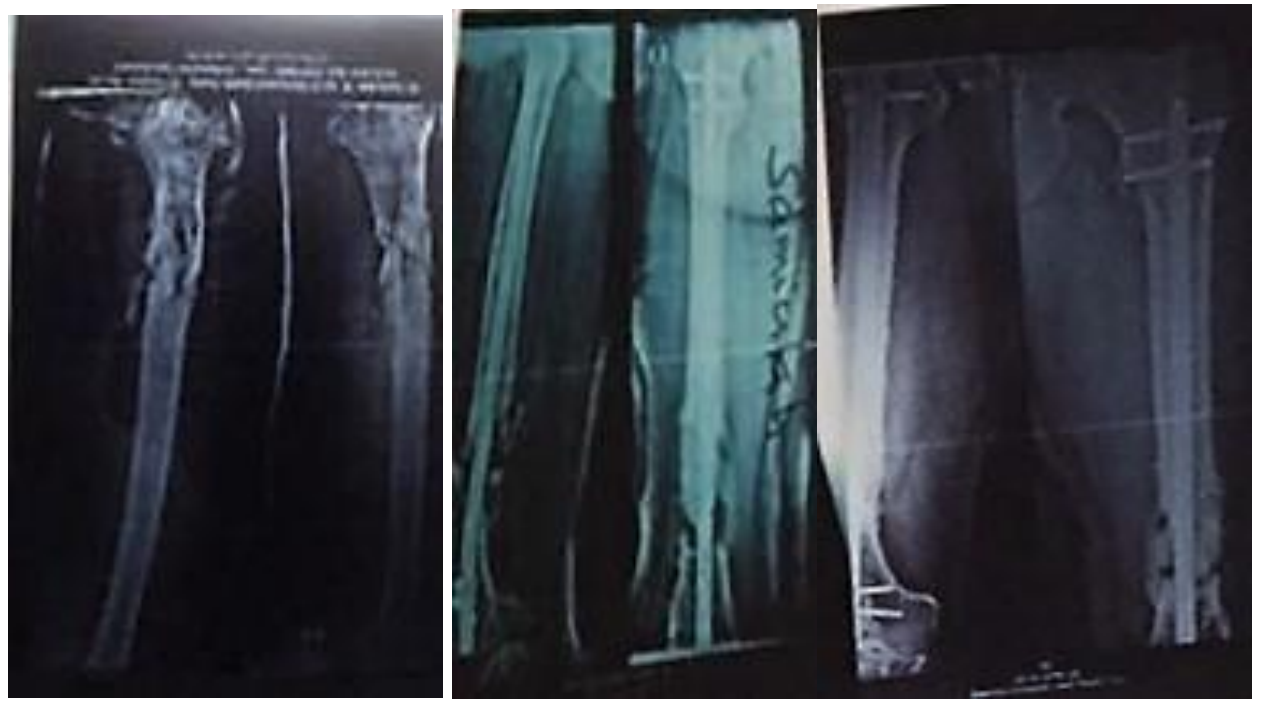

Figure 5. X-ray showing healing and improvement of infected focus, also in this infected non-union.

\section{DISCUSSION}

In the presence of serum proteins, even a small number of organisms can attach on to the implant surface and initiate a laconic and persistent proliferative response. The subsequent development of a biofilm inhibits ossteointegration and promote osteolysis the eventual loss of bone tissue provides space for the advancing biofilm and loss of attachment of implant. (7).

In our observation after putting cement in defect from loss of tissue in the femur, which has a bulk of, muscles from one side we provide a high level of antibiotic in the affected part, and from the other hand we prevent precipitation of protein over the nail, dead space is close to the exposed nail is occupied by cement (Figure 2).

Positive benefit from damaged part by providing high local antibiotic in the damaged part by putting antibiotic loaded cement, and prevention of blood collection at the site by space control with cement beads, and the ability to protect all healing factors which are released from the damaged bone by the trauma. All open fractures are by definition contaminated and must be treated in as such. The treatment methods may differ depending on the type of fracture. Infection risks also differ by fracture type and have been reported to be ranging from 0 to $2 \%$ for type I fractures,
2 to $10 \%$ for type II fractures, and 10 to $50 \%$ for type III fractures. $(8,9)$. But in our study we proposed the method of fixation which is comfortable for the patient, and has a higher rate of union (which is interlocking nail), which can be done easy in open wound reduction, and control dead space close to the damaged bone. Early fixation, the patient is in a good nutrition and immunity in the early days of trauma. All these were a reason of low infection rate in 2 from 35 gunshot wounds (6). Conversion from external fixation to an intramedullary nail has received considerable attention in the literature. Original reporting of this conversion had alarming results with infection and non-union rates of 44 and $50 \%$. Respectively (10). Subsequent studies have demonstrated better results. (11).

We observed improvement of osteomyelitis focus, and control of sinus infection in 15 infected non-union in 13 cases which were from previous infected implant, and clear improvement of bone quality between pre and post healing X-rays of those infected (which we think might be long term control of dead space by this solid nail which prevents scar and blood collection in the canal of the bone). Placement of antibiotic loaded cement over the fracture pin tract site which has close contact to the affected bone and nail. (12).

We noted a reactivation of infection after healing of the fractures in 2 infected non-union 
fractures, for this reason we advice the removal of cement in the infected fractures after 6 weeks, and delayed present open fractures, we advise to remove the cement at the time of nail removal.

\section{CONCLUSION}

The best way to deal with infection is to prevent it, while other factors as obesity, diabetes, and other co-morbidities increase the risk of infection. Surgical factors such as administration of peri-operative antibiotics, use of personal isolator suits and clean air rooms, efficient surgery, and copious irrigation can reduce the risk of infection.

In our method, we also provided local high concentration of antibiotic with the use of cement loaded antibiotic, and the control of dead space with bone defect and tissue loss. If we do not put cement or other solid material, then blood will definitely take space and that would be a media for infection.

\section{REFERENCES}

1. Hanssen AD: Local antibiotic delivery vehicles in the treatment of musculoskeletal infection. Clin Orthop Relate Res; 437:9196, 2005.

2. Joseph TN, Chen AL, Di Cesar PE; Use of antibiotic-impregnated cement in total joint replacement. $-j$ AM Acad Orthop Surg;11[1];38-47, 2003.

3. Deshmukh R.G., Thevarajan K., Kok C.S., Sivapathasundaram N., Geirge S.V.N. An intramedullary cement spacer in total hip arthroplasty. J arthroplasty.; 13(2): 197199, 1998.

4. Kuechle DK, Landon GC, Musher DM, Nobel PC: Elution of vancomycin, daptomycin, and amikacin from acrylic
AL-SADEK T., et al.

bone cement. Clin Orthop Relat Res; 264:302-308, 1991.

5. McLaren AC, Nelson CL, McLaren SG, DeClerk GR: The effect of glycine filler on the elution rate of gentamicin from acrylic bone cement: A pilot study. Clin Orthop Relat Res; 427:25-27, 2004.

6. Schoenfeld AJ, Goodman GP, Belmont PJ Jr: Characterization of combat-related spinal injuries sustained by a US Army Brigade Combat Team during Operation Iraqi Freedom. Spine J; 12(9): 771-776, 2012.

7. Javid Parvizi, MD, FRCS. Pouya Alijanipour, MD.Noreen J.Hickok, PhD. K. Scott Philips, PhD. Novel developments in the prevention, Diagnosis and treatment of Periprosthetic joint infections. $J A m$ Acad Ortho 2015.

8. Gustilo RB, Anderson JT. Prevention of infection in the treatment of one thousand and twenty-five open fractures of long bones: Retrospective and prospective analyses. J Bone Joint Surg Am.; 58:453-8, 1976.

9. Gustilo RB, Gruninger RP, Davis T. Classification of type III (severe) open fractures relative to treatment and results. Orthopedics; 10:1781-8, 1987.

10.McGraw JM, Lim EV. Treatment of open tibial-shaft fractures: External fixation and secondary intramedullary nailing. $J$ Bone Joint Surg Am.; 70:900-11, 1988.

11.Nowotarski PJ, Turen CH, Brumback RJ, Scarboro JM. Conversion of external fixation to intramedullary nailing for fractures of the shaft of the femur in multiply injured patients. $J$ Bone Joint Surg. Am.; 82:781-8, 2000.

12. Merritt K. Factors increasing the risk of infection in patients with open fractures. $J$ Trauma.; 28:823-7, 1988. 